\title{
Room temperature benzene gas detection using gated lateral BJT with assembled solvatochromic dye
}

\author{
Heng Yuan ${ }^{1}$, Bo Wang ${ }^{1}$, Se-Hyuk Yeom ${ }^{1}$, Dae-Hyuk Kwon ${ }^{2}$, Shin-Won Kang ${ }^{3}$ \\ 1 School of Electrical Engineering and Computer Science, Kyungpook National University 1370 \\ Sankyuk-dong, Bukgu, Daegu, Republic of Korea \\ ${ }^{2}$ Department of Electronic Engineering, Kyungil University, Gyeongsang, Republic of Korea \\ ${ }^{3}$ Department School of Electronics Engineering, College of IT engineering, Kyungpook National \\ University 1370 Sankyuk-dong, Bukgu, Daegu, Republic of Korea \\ swkang@knu.ac.kr
}

\begin{abstract}
In this study, a new type of room temperature operated volatile organic compounds (VOCs) gas sensor was developed based on gated lateral bipolar junction transistor (BJT). The proposed sensor device was fabricated using standard $0.35-\mu \mathrm{m}$ complementary metal-oxide-semiconductor transistor (CMOS) process. The specific metal-oxide-semiconductor field-effect transistor (MOSFET)-BJT hybrid structure cause that the device can be operated under room temperature without gate bias supply. 4amino-N-methylphthalimide (4-ANMP) dye is a kind of solvatochromic dye which has charge-transfer (CT) character was immobilized on the floating gate of the gated lateral BJT by using self-assembled monolayer (SAM) method, acted as the sensing membrane. Benzene gas as the sensing target was detected in this study. According to the results, the sensor device can be used for VOCs gas detecting at room temperature.
\end{abstract}

Key words: Volatile organic compounds (VOCs), gated lateral BJT, MOSFET-BJT hybrid, solvatochormic, charge transfer (CT), self-assembled monolayer (SAM)

\section{Introduction}

VOCs gases are given off from paint, rubbish or many kinds of articles for daily use, which give affection to human body. In the last decade, as we have become more aware of the need of a healthy environment, the demand of sensors for detecting pollutants or hazardous substances is on the increase. Besides, there are some reports shows that the VOCs concentration from exhaled breath of lung cancer patients, breast cancer patients, colorectal cancer patients, and prostate cancer patients are different from healthy people [1, 2]. Consequently, the VOCs detection has the significant not only environment monitoring but also provide basis to medical diagnosis. Many types of VOCs gas sensors have been reported and most of the sensor devices were opticaltype sensors which have high sensitive advantages and good selectivity properties. However, they have big size, high price, difficult to mass product, high power operation, need light source and detector disadvantages [3, 4]. The disadvantages can be overcome by microelectromechanical systems (MEMS) technology or complementary metal-oxide-semiconductor transistor (CMOS) technology. However, the disadvantage of high temperature operation cause them lose the low power advantage which the common MEMS and COMS technologies have $[5,6]$. In the previous works, we found the gated lateral bipolar junction transistor (BJT) can be used as sensor device which similar to metal-oxide-semiconductor field-effect transistor (MOSFET) based ionsensitive field-effect transistor (ISFET) [7]. Besides, we have illustrated that the gated lateral BJT can be used for protein biosensor and VOCs gas sensors using Reichardt's dye sensing membrane in room temperature conditions [8, 9]. However, the reliability was very poor because the charge transfer (CT) direction of Reichardt's dye was difficult to be controlled, which was coated on the surface of the device by spin coating method.

In this study, 4-amino-N-methylphthalimide (4ANMP) dye which is a kind of solvatochromic dye was used as the sensing membrane. Selfassembled monolayer (SAM) method was used for 4-ANMP dye sensing membrane fabrication. Then, benzene gas was detected by gated lateral BJT under room temperature condition. 
The result signal was detected by semiconductor parameter analyzer.

\section{Experiments and methods}

The gated lateral BJT was fabricated by 0.35 $\mu \mathrm{m}$ CMOS process which contains a lateral BJT, a vertical BJT, and a p-type MOSFET structures as shown in Fig. 1. The source and drain of MOSFET share with the emitter and collector of lateral BJT. Therefore, the emitter current of the proposed device contains lateral BJT current, vertical BJT current, and MOSFET channel current. Emitter was designed in the center of the device which can increase the efficiency of the BJT part. Gate, lateral collector, and base surrounded the emitter. According to the special structure, there are 3-types of operation method can be used, which are socalled BJT mode, MOSFET mode, and MOSFET-BJT hybrid mode. The operation methods can be explained as follows: First, the vertical BJT collector (substrate) and the lateral BJT collector were connected to ground as the reference potential. Then, supply a forwards bias to emitter, negative current to base, and a gate bias which bigger than threshold voltage ( $p$-type MOSFET) the device can operate in the BJT mode. If a forwards emitter bias, positive base current, and a gate bias which small than threshold voltage were supplied, the device can operate in the MOSFET mode. And if a forwards emitter bias, negative base current, and a gate bias which smaller than threshold voltage, the device can operate in the MOSFET-BJT hybrid mode.

At the Floating gate structure, which has the advantages of having a large sensing area and simple fabrication process, was used in this proposed device. Then sensing membrane was fabricated by SAM method which has much been used in biosensor technologies []. An approximately 30-nm-thick $\mathrm{Au}$ layer was deposited onto a $\mathrm{Si}_{3} \mathrm{~N}_{4}$ layer on floating gate using electron beam evaporator. In order to fabricate the sensing membrane, following materials were used: 11-mercaptoundecanoic acid (99\%, Sigma-Aldrich), NHydroxysuccinimide (NHS, Sigma-Aldrich), N(3-Dimethylaminopropyl)-N'-ethylcarbodiimide hydrochloride (EDC, Sigma-Aldrich), and 4ANMP dye (97\%, Sigma-Aldrich). First, a solution of 11-mercaptoundecanoic acid ( $1 \mathrm{mmol} / \mathrm{L}$ in ethanol solution) was applied to the floating gate of the gated lateral BJT for approximately $24 \mathrm{~h}$ to form the SAM. Next, a solution containing $50 \mathrm{mmol} / \mathrm{L} \mathrm{NHS,} 50 \mathrm{mmol} / \mathrm{L}$ EDC, and ethanol was applied to the floating gate of the gated lateral BJT for approximately $1 \mathrm{~h}$ to activate the SAM. Next, a $5 \mathrm{mmol} / \mathrm{L} 4-$ ANMP dye was applied to the gate of the device approximately $2 \mathrm{~h}$ at $50{ }^{\circ} \mathrm{C}$.

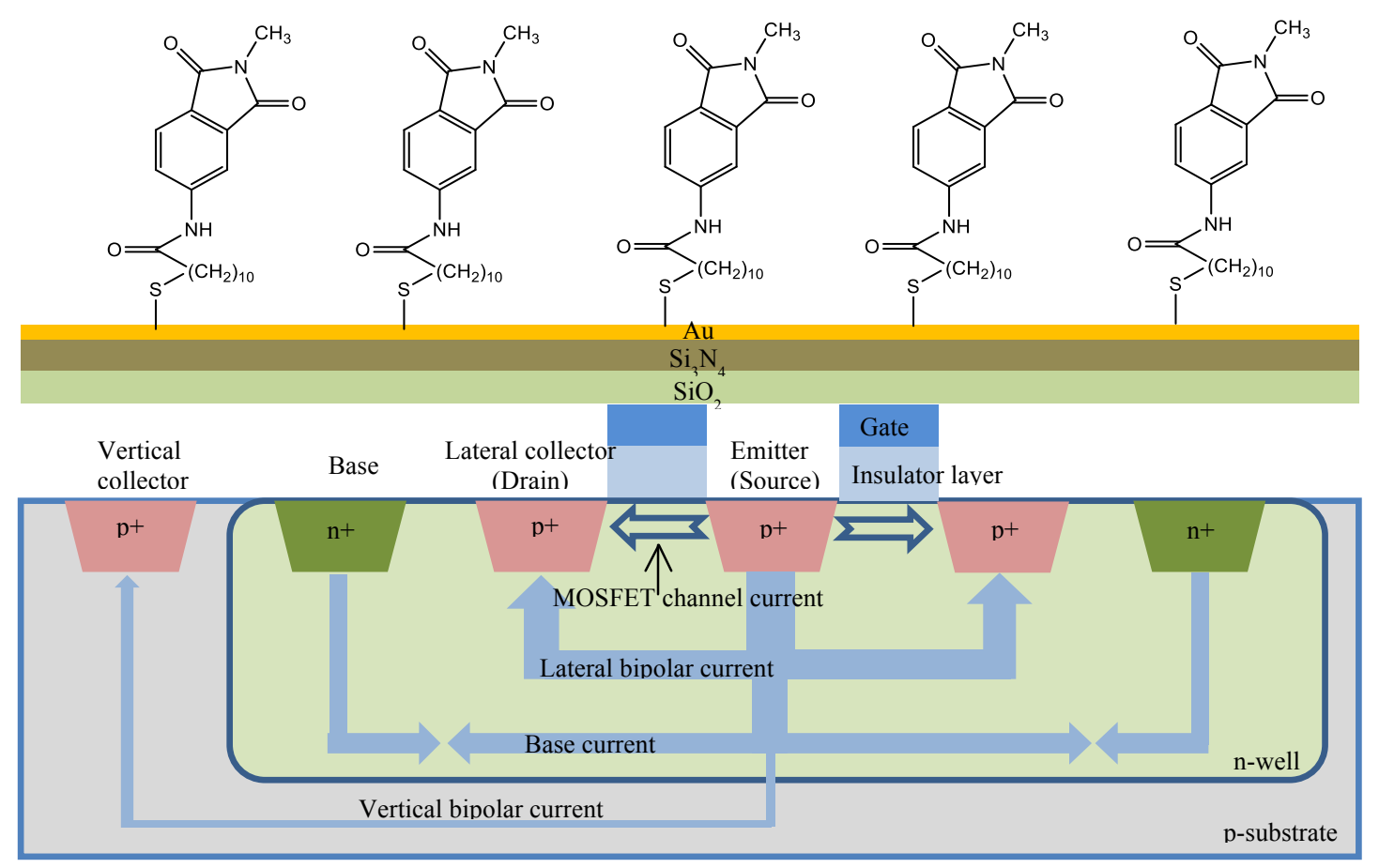

Fig. 1. Schematic of gated lateral BJT with 4-ANMP dye sensing membrane for VOC detection. 


\section{Results and discussions}

Before VOC gas detection, the sensing property of the gated lateral BJT was analyzed. In this experiment, gate bias was supplied directly. The result was shown in Fig. 2 which was also transconductance curve of device.

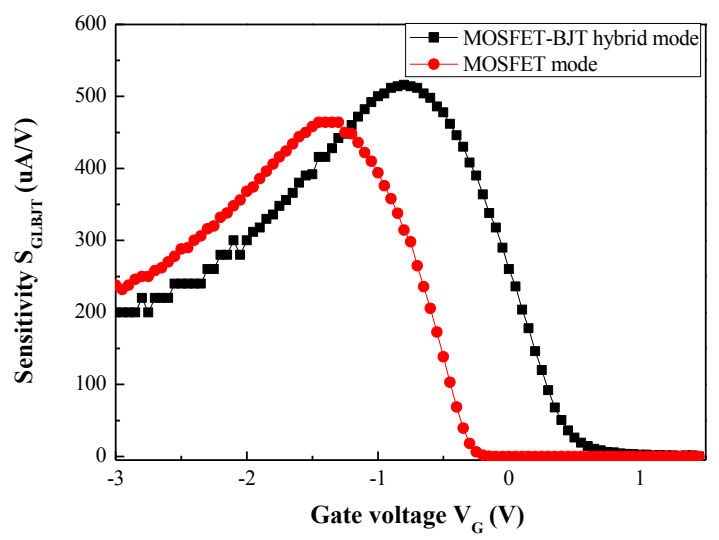

Fig. 2. Sensitivity property of MOSFET mode and MOSFET-BJT hybrid mode of gated lateral BJT.

The results indicated that in the MOSFET-BJT hybrid mode, the proposed device has sensitivity when gate bias was $0 \mathrm{~V}$ in room temperature condition. However, there was not any sensitivity in the MOSFET mode of the gated lateral BJT which similar as common MOSFET. The reason can be explained as follows: in the MOSFET-BJT hybrid, MOSFET part and lateral BJT part operate together. As gate bias increase positive, BJT effect become dominate and the sensitivity of MOSFET part decrease. On the other hand, it is different between the MOSFET channel current and lateral bipolar current. Therefore, there is a total current variation as the BJT effect increase. After the sensitivity of MOSFET part become small, the sensitivity of the gated lateral BJT mainly comes from the variation.

In this study, this property was used for VOC gas detection which gate bias cannot be supplied. The emitter bias was supplied $1 \mathrm{~V}$ and the base current was supplied $-10 \mu \mathrm{A}$. Benzene gas was detected by proposed gated lateral BJT with the concentration varied from $50 \mathrm{ppb}$ to $500 \mathrm{ppm}$. The time- $\mathrm{I}_{\mathrm{E}}$ curve was carried out for VOCs detection as shown in Fig. 3.

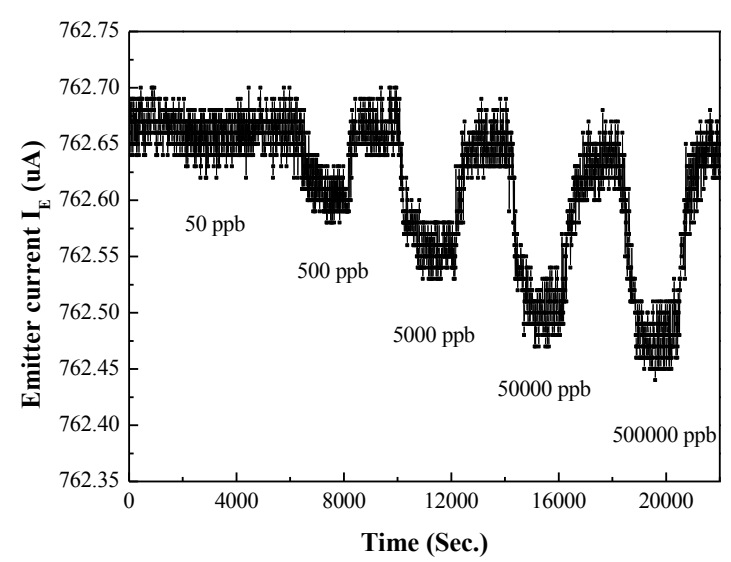

Fig. 3. Time-l $I_{E}$ curve results of benzene gas detection using gated lateral BJT.

According to the results, the emitter current of the proposed device were decreased as the concentration increased. The reason can be explained as follows: The reference condition was the $\mathrm{N}_{2}$ gas environment. After the VOC gas with the certain concentration which was synthetized by $\mathrm{VOC}$ gas and $\mathrm{N}_{2}$ gas, the polarity of the environment increased. Therefore, the $\pi^{*}-\pi$ type CT phenomenon of 4-ANMP dye appeared to response to the environment polarity changing [10]. Subsequently, the electron distribution in the molecule of 4-ANMP dye was changed. Overall, the electron distribution direction moved from the nodal point between SAM layer and 4ANMP dye to dye surface. In opposite, it is indicated that the potential of the floating gate of the device increased. Therefore, the channel current decreased. On the other hands, because the device was operated in the MOSFET-BJT hybrid mode, there was lateral BJT current in the emitter current. However, the BJT current has not any changes according to the base current invariable in this experiment. Therefore, the emitter current which contains MOSFET channel current and BJT current changing property follows MOSFET channel. As the concentration of the VOC gas increasing, the polarity of the environment increased. Hence, the results show that as the concentration increasing, the emitter current decreased.

Then, Fig. 3 was cleared up to concentration vs. emitter current decrement curve, as shown in Fig. 4. 


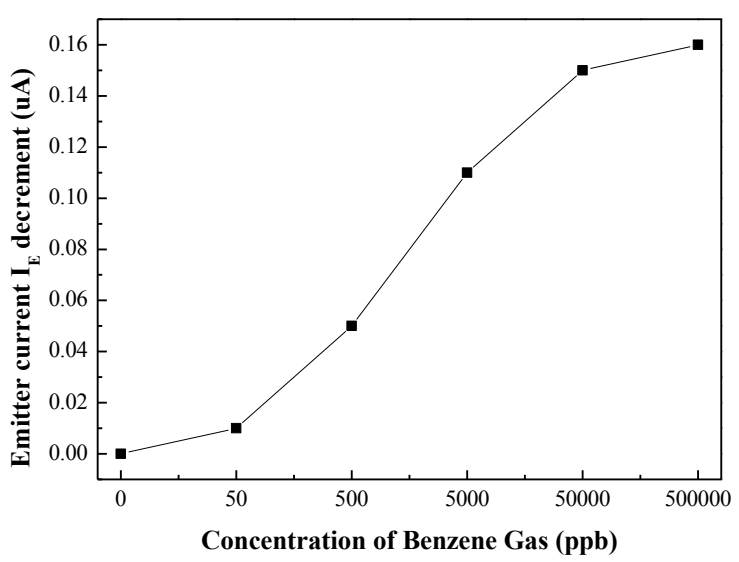

Fig. 4. Plots of benzene gas detection using gated lateral BJT. Graph of variable vs analyte concentration (style IMCS_Caption) for two different temperatures.

According to this figure, the minimum sensing limit of the proposed device for benzene gas detection was approximately $50 \mathrm{ppb}$. And when the concentration of benzene gas over $50 \mathrm{ppm}$, the sensor becomes to saturation state. The linear region of benzene gas detection using the gated lateral BJT was approximately from $50 \mathrm{ppb}$ to $50 \mathrm{ppm}$. And the sensitivity was approximately $0.05 \mu \mathrm{A} /$ decade.

Although the sensitivity was not very high, the proposed device shows the sensibility for benzene gas in the room temperature condition. In the future, several other VOCs gases will be detected and the sensitivity of the proposed device will be improved.

\section{Conclusion}

In this study, a novel benzene gas sensor based on gated lateral BJT was developed. The proposed device can operate under room temperature conditions which is a big advantage than other semiconductor based devices rely on the special structure. The device was fabricated by $0.35-\mu \mathrm{m}$ CMOS process which combines a MOSFET and a lateral BJT with both the emitter and source, and the collector and drain, coupled. MOSFETBJT hybrid mode, which is a kind of special operation method, was used in this study that can operate under room temperature conditions without gate bias supply. 4-ANMP dye which is a kind of solvatochromic dye has CT character was used as the sensing membrane to resolve the room temperature VOC gas detection problem. In order to improve the CT efficiency, 4-ANMP dye was ordered by SAM method. Benzene gas was used as the sensing target in this study. According to the results, the possibility of VOC gas detection using proposed device under room temperature was confirmed.

\section{Acknowledgements}

This work was supported by Basic Science Research program through the National Research Foundation of Korea (NRF) grant funded by the Korea government (MEST) (No. 2011-0001081). And this work was supported by Samsung Electronics Co., Ltd.

\section{References}

[1] G. Peng, U. Tisch, O. Adams, M. Hakim, N. Shehada, Y.Y. Broza, S. Billan, R. AbdahBortnyakk, A. Kuten, H. Haick, Diagnosing lung cancer in exhaled breath using gold nanopaticles, Nature Nanotechnology 4, 669-673 (2009); doi: 10.1038/NNANO.2009.235

[2] G. Peng, M. Hakim, Y.Y. Broza, S. Billan, R. Abdah-Bortnyak, A. Kuten, U. Tisch, H. Haick, Detection of lung, breast, colorectal, and prostate cancers from exhaled breath using a sigle array of nanosensors, British Journal of Cancer, 103, 542-551 (2010); doi: 10.1038/sj.bjc.6605810

[3] N.A. Rakow, K.S. Suslick, A colorimetric sensor array for odour visualization, Nature 406, 710-713 (2000); doi: 10.1038/35021028

[4] H. Yuan, S.H. Yeom, J.W. Lim, S. W. Kang, Sidepolished optical fiber order sensor for VOC detection based on solvatochromism, Sensor Letters 9, 87-91 (2011); doi: $10.1166 / \mathrm{s} 1.2011 .1425$

[5] M.Mori, H. Nishimura, Y. Itagaki, Y. Sadaoka, E. Traversa, Detection of sub-ppm level of VOCs based on a Pt/YSZ/Pt potentiometric oxygen sensor with reference air, Sensors and Actuators B 143, 56-61 (2009); doi: 10.1016/j.snb.2009.09.001

[6] S. Stegmeier, M. Fleischer, P. Hauptmann, Thermally activated platinum as VOC sensing material for work function type gas sensors, Sensors and Actuators B 144, 418-424 (2010); doi: 10.1016/j.snb.2009.02.021

[7] H.C. Kwon, D.H. Kwon, K.Sawada, S.W. Kang, The characteristics of $\mathrm{H}^{+}$-ion-sensivite transistor driving with MOS hybrid mode operation, IEEE Electron Device Letters 29, 1138-1141 (2008); doi: 10.1109/LED.2008.2002809

[8] H. Yuan, H.C. Kwon, S.H. Yeom, D.H. Kwon, S.W. Kang, MOSFET-BJT hybrid mode of the gated lateral bipolar junction transistor for $\mathrm{C}$ reactive protein detection, Biosensors and Bioelectronics 28, 434-437 (2011); doi: 10.1016/j.bios.2011.07.062

[9] H. Yuan, H.C. Kwon, S.H. Yeom, B. Wang, K.J. Kim, D.H. Kwon, S.W. Kang, Volatile organic compound gas sensor using a gated lateral bipolar junction transistor, Journal of Korean Physical Society 59, 478-481 (2011); doi: 10.3938/jkps.59.478

[10] C. Reichardt, Solvatochromic dyes as solvent polarity indicators, Chemical Reviews 94, 23192358 (1994); doi: 10.1021/cr00032a005 